\title{
Zernike Phase Plate Configuration at Intermediate Lens Position on JEM2200FS
}

\author{
Wah Chiu, Caroline J.-Y. Fu, Htet Khant and Sohei Motoki*
}

National Center for Macromolecular Imaging, Verna and Marrs McLean Department of Biochemistry and Molecular Biology, Baylor College of Medicine, Houston, TX 77030, USA *JEOL USA, Inc., 11 Dearborn Road, Peabody, MA 01960, USA

Cryo-electron microscopy is an emerging tool for structural biology to study biological specimens in their native conformational states. High contrast images with high signal-to-noise ratio are always beneficial for extracting features out of subcellular components in a cell tomogram or visualizing small macromolecules in single particle cryoEM. There are recent advances in enhancing image contrast using direct electron detectors and Zernike phase plate. The application of direct electron detector has demonstrated its usefulness in enhancing the low and high resolution contrast to facilitate the near atomic resolution structure determination of single particles. Zernike phase plate offers impressive low resolution image contrast to reveal structural details of subcellular components in a crowded environment of cell tomograms.

Zernike phase plate is made of a thin carbon film with a central hole, and is positioned at the focal plane of an objective lens. This configuration would change the modulation of the contrast transfer function from sine to cosine function. Therefore, the image contrast at low spatial frequencies is greatly enhanced. Implementation of Zernike phase plate has been done at the objective lens aperture position without making extensive modification to an existing microscope. We have used such configuration to collect data on a number of biological systems $[1,2]$. Alternatively, one can place the phase plate at a position conjugate to the back focal plane of an objective lens. For instance, in JEM-2200FS, one can place the phase plate at the focal plane of the objective mini lens, which is at the selected area (SA) aperture position (Figure 1). In this configuration, the effective focal length for the phase plate is doubled $(\mathrm{f}=6.3 \mathrm{~mm}$, which is about twice that of an objective lens).

The extent of image contrast enhancement depends on the cut-on frequency of the Zernike phase plate, which is inversely proportional to the hole diameter and directly proportional to the effective focal length of the lens (Figure 2). Placing the phase plate at the SA position would enhance the contrast of the images when using the same hole size as most of the phase plates currently available as well as allow the objective aperture to be used simultaneously. In order to use such phase plate configuration in low dose operation, we have to implement an imaging protocol for search, focus and photo mode different from the conventional one. For example, projector deflector coils instead of image shift coils are used for off-axis focusing. A preliminary test with a number of frozen hydrated biological specimens shows promising results. A tomographic data collection and reconstruction of mammalian cells are being presented to illustrate its general applicability.

Acknowledgement: This research has been supported by a NIH grant (P41GM103832).

References 
[1] Danev, R \& Nagayama, K (2008) Single particle analysis based on Zernike phase contrast transmission electron microscopy. J Struct Biol 161(2):211-218.

[2] Dai, W, Fu, C, Raytcheva, D, Flanagan, J, Khant, HA, Liu, X, Rochat, RH, HaasePettingell, C, Piret, J, Ludtke, SJ, Nagayama, K, Schmid, MF, King, JA, \& Chiu, W (2013) Visualizing virus assembly intermediates inside marine cyanobacteria. Nature 502(7473): 707-710.

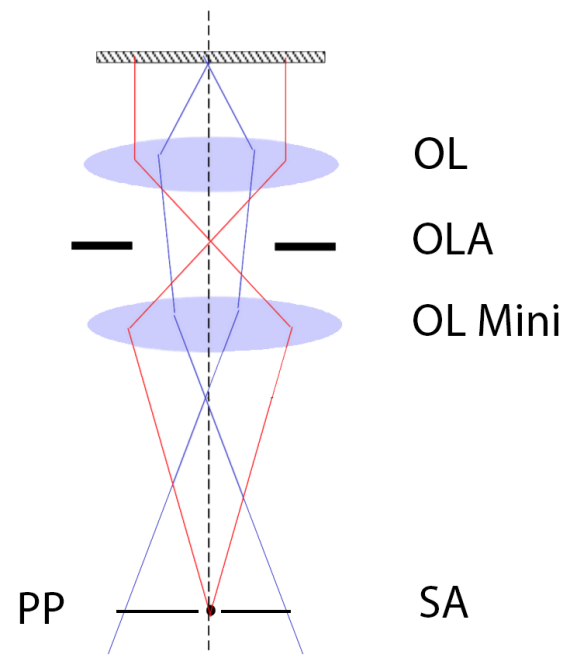

Fig 1: Electron optical ray diagram for phase plates (PP) located at selected area aperture (SA). OL, OLA and OL Mini stand for objective lens, objective lens aperture and objective mini lens respectively.

$$
\text { cut-on periodicity }=\frac{f_{o} \lambda}{d / 2} \quad \begin{aligned}
& f_{0}: \text { focal length of objective } \\
& \lambda: \text { wavelength of electron wave } \\
& d \text { : diameter of a central hole }
\end{aligned}
$$

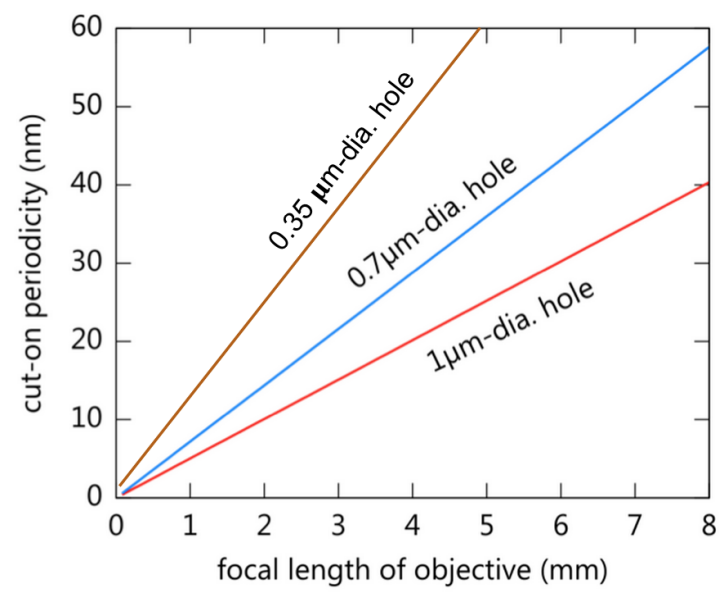

Fig 2: Relationship between cut-on periodicity, phase plate hole diameter and objective lens focal length. 